\section{Exploring molecular isotopic variation in intact oxyanions by Electrospray- Orbitrap mass spectrometry}

CAJ NEUBAUER ${ }^{1}$, ANDREAS HILKERT ${ }^{2}$, J.K. BÖHLKE ${ }^{3}$, XINGCHEN TONY WANG ${ }^{4}$ AND SEBASTIAN KOPF ${ }^{1}$

${ }^{1}$ University of Colorado Boulder

${ }^{2}$ Thermo Fisher Scientific

${ }^{3}$ United States Geological Survey

${ }^{4}$ Boston College

Presenting Author: 123caj@gmail.com

Electrospray-Orbitrap mass spectrometry (MS) is a new tool to measure isotopic abundances of solutes, including oxyanions such as sulfate, nitrate, phosphate, and others. This technique allows convenient access to intact polyatomic ions. Because the measurement preserves the native bonding of isotopes in a molecule it provides an intrinsically multi-elemental and multidimensional isotopic readout. For example, seven intact isotopologs of nitrate can be quantified simultaneously (see Figure; unsubstituted base peak not shown). The spectra provide multiple alternative paths for calculating $\delta^{15} \mathrm{~N}, \delta^{18} \mathrm{O}$, and $\delta^{17} \mathrm{O}$ values, as well as opportunities to explore non-statistical isotopic distributions (i.e. anti/clumped). The technology development is now reaching a level of precision that could reveal significant isotopolog anomalies that are complementary to conventional triple oxygen isotope systematics.

In this presentation we will provide an overview of ongoing efforts in developing Electrospray-Orbitrap as an accurate and precise isotope-ratio MS. The focus will be on oxyanions that are relevant throughout a wide suite of ancient and modern environmental studies. We will introduce new methodologies using nitrate as a simple model system to illustrate isotopic measurement principles; present a basic theoretical framework for interpreting isotopolog patterns; and summarize provisional validations of the method by comparisons to independent data for nitrate and sulfate. We will then focus on exploring new isotopolog anomalies in environmental samples that are related to triple isotope systematics. Our goal here is to facilitate joint learning and problem solving on this emerging frontier by discussing how recent progress can be used to catalyze new triple oxygen isotope studies for hypothesis testing.

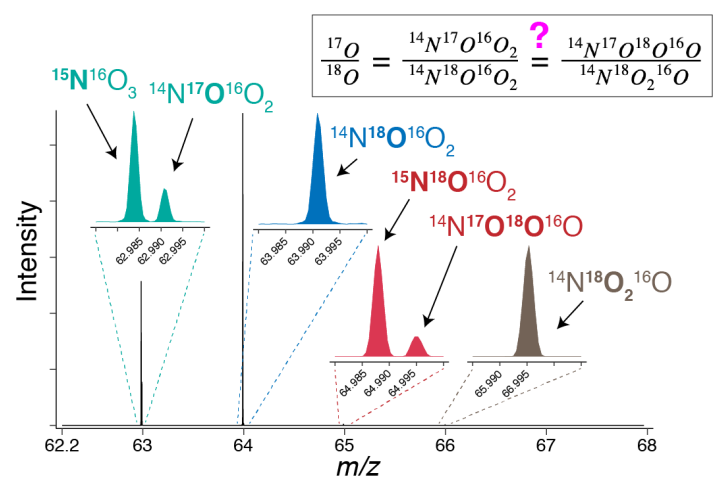

